\title{
Subject Index vol. 24
}

compiled by $\lambda$ Vilhelm Baumann, Zurich

Acute renal failure, haemodialysis by $\Lambda$ orta-Cava catheter, 30

Adrenal medulla, electron microscopic study, 79

Anurie oder mechanisches Abflusshin-dernis, 139

Anuria renal u obstruccíón mecánica, 139

Aorta-cava Teflon catheter for haemodialysis, 30

Beckenvenendarstellung, 551 Biopsie de la prostate par aspiration, 560 Bleeding in prostatectomy, 418, 426 Blutstillung bei Prostatektomie, 418, 426

Calcilithiasis, 318

Cavernography, 551

Cístectomia, 193

Competitive protein binding, 481

Congelation de la prostate, 145, 153, 160, 177

Congenital reflux, surgical management, 502

Congenital reflux, the etiology, 119

Cryotherapy of heterotransplanted human cancer, 166

Cryosurgery, 439

Cryosurgery of the prostate, $145,153,160,177$

Denis Browne's hypospadias correction,

135 Derivación urinaria temporal en las in-

tervenciones sobre uretra, 97

Einzelniere, chirurgische Behandlung,

527 Electromanometry, 289 Electromyography, 289 Epididyme, Malakoplakie, 364

Female urethra, reconstruction, 73 Feocromocitoma, estudio mediante mi-

croscopio electronico, 79 Fibrinolytische Haemorrhagie, 385

Haemodialysis, aorta-cava Teflon catheter, 30 Harnableitung bei Operationen, 97 Harnblase, Aetiologie der Tumoren, 276 -, Rekonstruktion, 193 •-, Transplantation im Tierexperiment, 193 Harnröhre, Wiederherstellung bei der

Frau, 73 Hemorrhage by fibrinolysis, 385 Hémostase dans la prostatectomie, 418,

426 Hibernation, action du Mannitol, 45 Hyperparathyroidisme primaire, 466 Hypertension, renovascular, 1, 12 Hypertonie, renovaskuläre, 1, 12 Hypospadie-Korrektur nach Denis

Browne, Fistelverschluss, 135 Hippuran-]-131 renography, 37

Intranatal renal rupture, 58

Kältebehandlung heterotransplantierter Tumoren, 166

Subject Index vol. 24

571

Kidney, hypertrophied, 300 -, solitary, surgical treatment, 527 Kryotherapie heterotransplantierter menschlicher Tumoren, 166 
Lithiases calciques, 318 Lysozymuria in renal calculosis, 310

Malakoplakia testis et epididymidis, 364 Malakoplakia vesicae urinariae, 355 Mannitol treatment with hibernation, 45 Megaloureter congenitalis, 344 Méga-uretère primaire, Гétiologie, 119 -, traitement chirurgical, 502

Nebennierenmark, elektronenmikroskopische Untersuchung, 79 Néphrogrammes par Híppuran-I-131, 37 Nephrolithiasis, Calcilithiasis, 318 -, Lysozymuria, 310 Nieren, kompensatorisch hypertrophe, 300 Nierenarterienstenose und Hypertonie,

1, 12 Nierenruptur, geburtstraumatische, 58 Nierenversagen. infolge Vergiftung, Ill -, Hämodialyse durch Aorten-Cava Ka-

theter, 30

Obstruktive Uropathie oder Anurie, 139 Oxalate calculosis, 318

Pelvic phlebography, 551 Perineale Harnfistel, 97 Phlébographie pelvienne, 551

Pheochromocytoma, an electron microscopic study, 79 Phosphate metabolism, 66 Primary megaureter, surgical management, 502 Prostata, Aspirationsbiopsie, 560 Prostataoperationen, Kältechirurgie, 145, 153, 160, 177 Prostate, cryochirurgie, 145, 153, 160, 177 Prostatektomie, Blutstillung, 418, 426 Prostatic carcinoma, biopsy technic, 560 Prostatic freezing, 145, 153, 160, 177 Protein binding, competitive, 481

Pyelo-Ureteroscopy, 289 Pyelonephritis xanthogranulomatosa, 330 Pyrophosphatstoffwechsel, 66

Rein, hypertrophie compensatrice, 300 -, insuffisance aiguë, hémodialyse par sondes transfémorales, 30

- $\quad$ intoxiqué, la recuperation fonction-

nelle, Ill

-, rupture par traumatisme obstetrical,

58 -, sténose artérielle et hypertension, 1 ,

12

- $\quad$ unique, traitement chirurgical, 527

Reflux congenital, $\Gamma$ étiologie, 119

-, traitement chirurgical, 502 -, sterile, 460

Renal artery stenosis and hypertension, 1,24

calculosis, Lysozymuria, 310

failure, poisonous, Ill

rupture, intranatal, 58 Renography, Hippuran-I-131, 37 Renovascular hypertension, 1, 12 Riñón, actividad pirofosfatasa, 66 -, estenosís de la arteria, 1, 12

-, íallo agudo, hemodiálisis repetidas, 30 -, rehabilitación del fallo por intoxica-

ción, Ill -, rupturas obstetricas, 58

Solitary kidney, surgical treatment, 527 Spermatogenesis in the rat, 372 Suprapubische

Cystostomie, 97 Surrénale, etude électro-microscopique, 79

Testicule, malakoplakia, 364 Testosterone metabolism in man over 60

years, 481 Trigonal cystectomy, 193 Testosteronspiegel im Plasma, 481

Untersuchungsmethoden, urodynami-sche, 289

Urethral surgery, temporary urinary diversion, 97

Uretra femenina, reparación, 73 
Urètre, derivation temporaire de l'urine, 97

572 Subject

Urinary bladder, aetiology of tumours,

276 -, experimental transplantation, 193 -, malakoplakia, 355 -, reconstruction, 193

Urodynamische Untersuchungsmetho-

den, 289 Uromanometry, 289

Vejiga urinaria, etiología de los tumores,

276 -, malakoplakia, 355

Index vol. 24

Vejiga, reconstucción, 193

-, trasplante en experimentación animal, 193

Vereisung der Prostata, 145, 153, 160, 177

Vessie urinaire, étiologie des tumeurs, 276

-, malakoplakie, 355

-, reconstruction, 193

-, transplantation chez Tanimal, 193

Xanthogranulomatous pyelonephritis, 330 